\title{
Identification and Classification of Alzheimer's Disease Patients Using Novel Fractional Motion Model
}

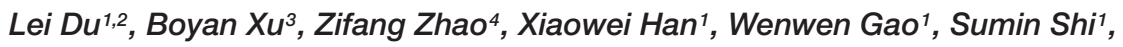 \\ Xiuxiu Liu' ${ }^{1}$, Yue Chen ${ }^{1}$, Yige Wang', Shilong Sun ${ }^{1}$, Lu Zhang ${ }^{5}$, Jiahong Gao ${ }^{6,7,8}$ and \\ Guolin $\mathrm{Ma}^{1,2 *}$
}

\begin{abstract}
${ }^{1}$ Department of Radiology, China-Japan Friendship Hospital, Beijing, China, ${ }^{2}$ Graduate School of Peking Union Medical College, Chinese Academy of Medical Sciences and Peking Union Medical College, Beijing, China, ${ }^{3}$ Beijing Intelligent Brain Cloud Inc., Beijing, China, ${ }^{4}$ Department of Anesthesiology, Peking University First Hospital, Peking University, Beijing, China, ${ }^{5}$ Department of Science and Education, Shangluo Central Hospital, Shangluo, China, ${ }^{6}$ Beijing City Key Lab for Medical Physics and Engineering, Institute of Heavy Ion Physics, School of Physics, Peking University, Beijing, China, ${ }^{7}$ Center for MRI Research, Academy for Advanced Interdisciplinary Studies, Peking University, Beijing, China, ${ }^{8}$ McGovern Institute for Brain Research, Peking University, Beijing, China
\end{abstract}

\section{OPEN ACCESS}

Edited by:

Danny J. J. Wang,

University of Southern California, Los Angeles, United States

Reviewed by:

Arvind Caprihan,

Mind Research Network (MRN),

United States

Yong Fan,

University of Pennsylvania,

United States

*Correspondence:

Guolin Ma

maguolin1007@qq.com

Specialty section:

This article was submitted to

Brain Imaging Methods,

a section of the journal

Frontiers in Neuroscience

Received: 07 May 2020

Accepted: 30 June 2020

Published: 17 September 2020

Citation:

Du L, Xu B, Zhao Z, Han X, Gao W, Shi S, Liu X, Chen Y, Wang Y,

Sun S, Zhang L, Gao J and Ma G (2020) Identification and Classification of Alzheimer's Disease Patients Using

Novel Fractional Motion Model.

Front. Neurosci. 14:767.

doi: 10.3389/fnins.2020.00767
Most diffusion magnetic resonance imaging (dMRI) techniques use the monoexponential model to describe the diffusion process of water in the brain. However, the observed dMRI signal decay curve deviates from the mono-exponential form. To solve this problem, the fractional motion (FM) model has been developed, which is regarded as a more appropriate model for describing the complex diffusion process in brain tissue. It is still unclear in the identification and classification of Alzheimer's disease (AD) patients using the FM model. The purpose of this study was to investigate the potential feasibility of FM model for differentiating $A D$ patients from healthy controls and grading patients with $A D$. Twenty-four patients with $A D$ and 11 healthy controls were included. The left and right hippocampus were selected as regions of interest (ROls). The apparent diffusion coefficient (ADC) values and FM-related parameters, including the Noah exponent $(\alpha)$, the Hurst exponent $(H)$, and the memory parameter $(\mu=H-1 / \alpha)$, were calculated and compared between AD patients and healthy controls and between mild $\mathrm{AD}$ and moderate $\mathrm{AD}$ patients using a two-sample $t$-test. The correlations between FM-related parameters $\alpha, H, \mu$, and ADC values and the cognitive functions assessed by mini-mental state examination (MMSE) and Montreal cognitive assessment (MoCA) scales were investigated using Pearson partial correlation analysis in patients with $A D$. The receiver-operating characteristic analysis was used to assess the differential performance. We found that the FM-related parameter $\alpha$ could be used to distinguish AD patients from healthy controls $(P<0.05)$ with greater sensitivity and specificity (left $\mathrm{ROI}, 0.917$ and 0.636 ; right $\mathrm{ROI}, 0.917$ and 0.727$)$ and grade $\mathrm{AD}$ patients $(P<0.05)$ showed higher sensitivity and specificity (right ROI, 0.917, 0.75). The $\alpha$ was found to be positively correlated with MMSE $(P<0.05)$ and MoCA $(P<0.05)$ scores in patients with $\mathrm{AD}$, indicating that the $\alpha$ values in the bilateral hippocampus were a potential MRI-based biomarker of disease severity in AD patients. This novel diffusion model may be useful for further understanding neuropathologic changes in patients with AD.

Keywords: diffusion magnetic resonance imaging, fractional motion model, anomalous diffusion, Alzheimer's disease, hippocampus 


\section{INTRODUCTION}

Alzheimer's disease (AD) is the most common neurodegenerative disease and is characterized by memory loss and cognitive decline (Reddy and Oliver, 2019). According to the World Health Organization (WHO), dementia affects nearly 47.5 million individuals worldwide and still increases by approximately 7.7 million new cases each year (Khan et al., 2017; Salvatore et al., 2018). As the most common type of dementia, AD may account for 60-70\% of these cases (Wortmann, 2012; Alzheimer's Association, 2016; Khan et al., 2017) and has a significant impact on the life quality of patients and societal costs. The pathogenesis of $\mathrm{AD}$ is extremely complicated, mainly including the deposition of amyloid- $\beta(A \beta)$ and hyperphosphorylation of tau protein, which results in the formation of $A \beta$-plaques and intracellular neurofibrillary tangles (NFTs) separately (Kidd, 1963; Hyman et al., 1984; Braak and Braak, 1991; Wegmann et al., 2010; Aisen et al., 2017), then causes neuronal death. Now the diagnosis of $\mathrm{AD}$ is complicated and the accuracy is difficult. Therefore, it is of great significance to develop an effective diagnostic method for $\mathrm{AD}$ in clinical research (Cummings, 2017).

Diffusion magnetic resonance imaging (dMRI) is a powerful and non-invasive tool that can describe the random motion of water molecules in biological tissues, provide unique information about the microscopic properties, and is highly sensitive to detecting changes in gray and white matter in the brain (Yoshiura et al., 2003). The diffusion process of water molecules in the nervous system is directionally dependent (Chenevert et al., 1990; Moseley et al., 1990). And this directional dependence, namely anisotropy, occurs primarily due to the inherent axonal membranes that hamper water molecule diffusion and the dense packing of axons (Beaulieu, 2002). Measurements of the anisotropy of water molecules diffusion at the micron level reflect the changes of the underlying microstructure.

Compared with other magnetic resonance imaging (MRI) modalities, dMRI investigates the diffusion process at the cellular scale in tissues (e.g., micrometers), which is far superior to the typical millimetric image resolution (Le Bihan and JohansenBerg, 2012). At present, one of the most widely used dMRI techniques in clinical application is the apparent diffusion coefficient (ADC), and $b$-values obtained by dMRI frequently range from 0 to $1000 \mathrm{~s} / \mathrm{mm}^{2}$. Apparent diffusion coefficient is widely used in clinical practice, and it can be used to differentiate brain tumors (Yamasaki et al., 2005) and grade tumors (Bulakbasi et al., 2004), and distinguish mild cognitive impairment (MCI) and AD (Pietroboni et al., 2018; Xue et al., 2019). Diffusion tensor imaging (DTI) is another widely used dMRI technique in the research. The fractional anisotropy (FA) and mean diffusivity (MD) obtained from DTI are potential biomarkers of brain abnormalities in patients with $\mathrm{MCI}$ and $\mathrm{AD}$ (Bosch et al., 2012; Nir et al., 2013; Mayo et al., 2018). However, both ADC and DTI have some limitations. Firstly, it is well known that conventional dMRI uses a mono-exponential model and assumes a standard diffusion process in biological tissues. However, many studies have found that the observed dMRI signal decay curve deviates from the mono-exponential form (De Santis et al., 2011). Both ADC and DTI are based on the standard monoexponential diffusion model, and the observed diffusion-time reflects the non-Gaussian nature of diffusion (Fieremans et al., 2016; Xu et al., 2017a). Secondly, the tensor model of DTI is too simple, which means that its indices can be affected by several features of the microstructure. To solve this problem, many models based on different theories and anomalous diffusion processes have been developed to find the optimal agreement between the experimentally observed signal decay curve and the proposed fitting curves (Mulkern et al., 1999; Maier et al., 2001; Jensen et al., 2005; Magin et al., 2008). These models guarantee a more detailed detection of the differences between disease types and disease grades but their signal decay curves are still different.

Recently, a novel fractional motion (FM) model was developed by the Center for MRI Research at Peking University. The FM model is regarded as a more appropriate model in the biophysics community for describing the complex diffusion process in biological systems (Magdziarz et al., 2009; Burnecki and Weron, 2010; Weiss, 2013), and it is a promising model for describing the diffusion process of brain tissue. Several studies have demonstrated that the FM model is a better model to explain the diffusion process of biological living cells (Magdziarz et al., 2009). The FM model assumes that the diffusion process of tissues is $H$-self-similar, $\alpha$-stable, and has stationary increments (Xu et al., 2017b). The symbol $\alpha$ is the Noah exponent that can quantify the fluctuations of the random process. When $\alpha=2$, the increments are Gaussian distributed, while when $0<\alpha<2$, the increments are Lévy distributed (Xu et al., 2017b; Xu et al., 2017a). $H$ is the Hurst exponent, which depicts the self-similarity property of molecular trajectories. $\mu$ is the memory parameter and $\mu=H-1 / \alpha$. When $\mu>0$, the increments are positively correlated and show long-range dependence (long memory, persistence), while when $\mu<0$, the increments of the process are negatively correlated and show short-range dependence (short memory, anti-persistence) (Xu et al., 2017a,b).

Previous studies have found that the FM-related parameter maps of healthy people showed obvious contrasts among normal brain tissues (Fan and Gao, 2015), and the FM-related parameter maps are superior to ADC in differentiating between low-grade and high-grade gliomas (Xu et al., 2017b, 2018). However, the parameters $(\alpha, H$, and $\mu)$ of the FM model in identifying $\mathrm{AD}$ patients from healthy controls and grading $\mathrm{AD}$ patients is still not clear. Therefore, the purpose of this study was to investigate the potential feasibility of the FM model for distinguishing $\mathrm{AD}$ patients from healthy controls and grading $\mathrm{AD}$ patients.

\section{MATERIALS AND METHODS}

\section{Subjects}

This study was approved by the ethics committee of ChinaJapan Friendship Hospital and informed consent was obtained from all subjects. The cognitive function of all participants was 
assessed using the mini-mental state examination (MMSE) scale and Montreal cognitive assessment (MoCA) scale. A total of 24 $\mathrm{AD}$ patients and 11 healthy controls underwent MRI examination and MMSE and MoCA scale assessment. These AD patients visited China-Japan Friendship Hospital between November 2015 and March 2019. The clinical diagnosis of AD met criteria as determined by the National Institute of Neurological and Communicative Disorders and Stroke and the Alzheimer's Disease and Related Disorders Association (NINCDS-ADRDA) (1984) (Du et al., 2018). Patients met the following criteria: (a) The MR image quality was good, no artifacts; (b) patients had no concurrent brain diseases; and (c) patients had no limb activity disorders, aphasia, visual, and hearing impairment. Healthy controls were recruited from the local community. Healthy controls with a history of cardiovascular, neurologic, metabolic, and psychiatric disorders or brain abnormalities detected by conventional MRI were excluded from this study, and the MMSE scores of healthy control were between 26 and 30. Table 1 shows their clinical characteristics. All participants underwent conventional MRI, 3D T1weighted imaging, and dMRI.

\section{Image Acquisition}

Brain MR imaging was acquired on a 3.0 Tesla (T) MRI scanner (GE Healthcare, Discovery MR750, United States) with an eightchannel head coil. Using a special Stejskal-Tanner single-shot spin-echo echo-planar-imaging sequence to obtain dMRI images of all subjects.

We did not fix the diffusion gradient separation time $(\Delta)$ in the process of scanning the conventional dMRI sequence in order to fit the FM model. Specifically, $\Delta$ was arrayed at 27.060, 39.560 , and $52.060 \mathrm{~ms}$. The diffusion gradient amplitude $\left(\mathrm{G}_{0}\right)$ was arranged as $15.67,19.68,24.73,31.06,39.01$, and $49.00 \mathrm{mT} / \mathrm{m}$ for each $\Delta$ value, which were selected to be approximately evenly spaced on the log axis. The gradient duration constant ( $\delta$ ) was fixed at $20.676 \mathrm{~ms}$. So, we obtained 18 non-zero b-values $\left(151,239,377,595,939,1481 \mathrm{~s} / \mathrm{mm}^{2}\right.$ for $\Delta$ at $27.060 \mathrm{~ms}, 245$, $387,611,964,1521,2399 \mathrm{~s} / \mathrm{mm}^{2}$ for $\Delta$ at $39.560 \mathrm{~ms}$, and 339 , $535,845,1333,2103$, and $3317 \mathrm{~s} / \mathrm{mm}^{2}$ for $\Delta$ at $52.060 \mathrm{~ms}$ ) in each gradient direction, respectively. To minimize the effect of diffusion anisotropy, we applied the diffusion gradients in three

TABLE 1 | Demographic information and clinical assessment scores for all subjects.

\begin{tabular}{lcccc}
\hline & \multicolumn{2}{c}{ AD patients } & & \\
\cline { 2 - 4 } & Mild AD & Moderate AD & $\begin{array}{l}\text { Healthy } \\
\text { controls }\end{array}$ & P-value \\
\hline Number & 12 & 12 & 11 & - \\
Male/female & $6 / 6$ & $3 / 9$ & $2 / 9$ & $>0.05$ \\
Age & $65.83 \pm 10.06$ & $72.08 \pm 3.75$ & $65.27 \pm 6.60$ & $>0.05$ \\
Education & $13.42 \pm 3.06$ & $10.50 \pm 3.87$ & $10.64 \pm 3.30$ & $>0.05$ \\
MMSE score & $23.17 \pm 11.27$ & $19.08 \pm 1.44$ & $28.82 \pm 1.08$ & $<0.05$ \\
MoCA score & $19.50 \pm 2.39$ & $16.50 \pm 2.15$ & - & -
\end{tabular}

$\overline{A D}$, Alzheimer's disease; MMSE, mini-mental state examination; MoCA, Montreal cognitive assessment. orthogonal directions (the $x$-axis, $y$-axis, and $z$-axis). Moreover, a total of 12 images with $b=0$ were obtained.

The dMRI sequence parameters were repetition time $(\mathrm{TR})=3800 \mathrm{~ms}$; echo time $(\mathrm{TE})=110 \mathrm{~ms}$; flip angle $(\mathrm{FA})=90^{\circ}$; number of excitations $=2$; accelerating factor $=2$; field-ofview $($ FOV $)=240 \mathrm{~mm} \times 240 \mathrm{~mm}$; matrix size $=128 \times 128$; slice thickness $=5.0 \mathrm{~mm}$; number of slices $=27$; and voxel size $=1.875 \mathrm{~mm} \times 1.875 \mathrm{~mm} \times 5 \mathrm{~mm}$. Since high in-plane resolution was preferable, a large slice thickness had to be chosen in order to achieve an adequate SNR. The total scan time was $8 \mathrm{~min} 33 \mathrm{~s}$. A T1w MRI was acquired in sagittal plane and the parameters were $\mathrm{TR}=6.7 \mathrm{~ms}$; TE $=$ Min Full; $\mathrm{FA}=12^{\circ}$; slice thickness $=1.0 \mathrm{~mm}$; number of slices $=192$; FOV $=256 \mathrm{~mm} \times 256 \mathrm{~mm}$; matrix size $=256 \times 256$; and scan time $=4 \min 10 \mathrm{~s}$.

\section{Image Segmentation}

The left and right hippocampus were selected as the regions of interest (ROIs) in the present study (Figure 1). Firstly, the ROIs were drawn manually by an experienced radiologist (Lei $\mathrm{Du}, 5$ years working experience) on the 3D T1 weighted images using MRICRON, then the ROI on T1w MRI was co-registered to diffusion $\mathrm{MR}$ imaging to improve the accuracy of the hippocampal outline. In all subjects, the ROIs' location was segmented and excluded ambiguous voxels.

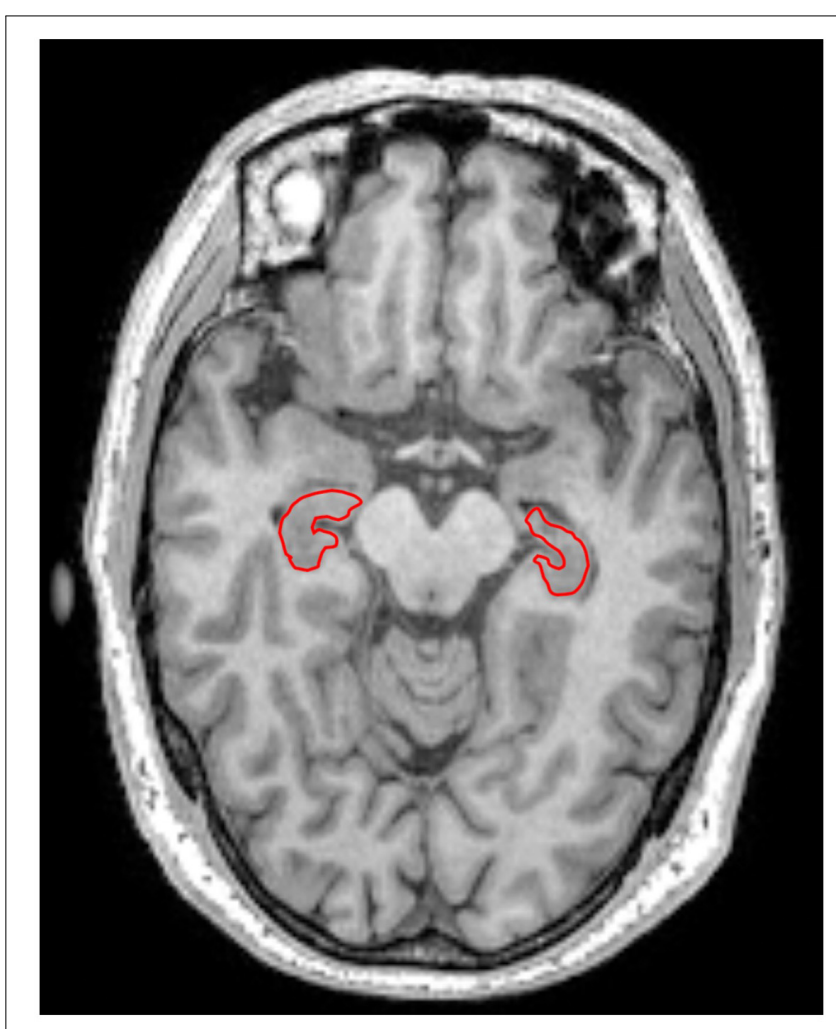

FIGURE 1 | Left and right hippocampus were selected as regions of interest (ROIs) in this study. ROls were encircled in red line in T1 weighted imaging. 


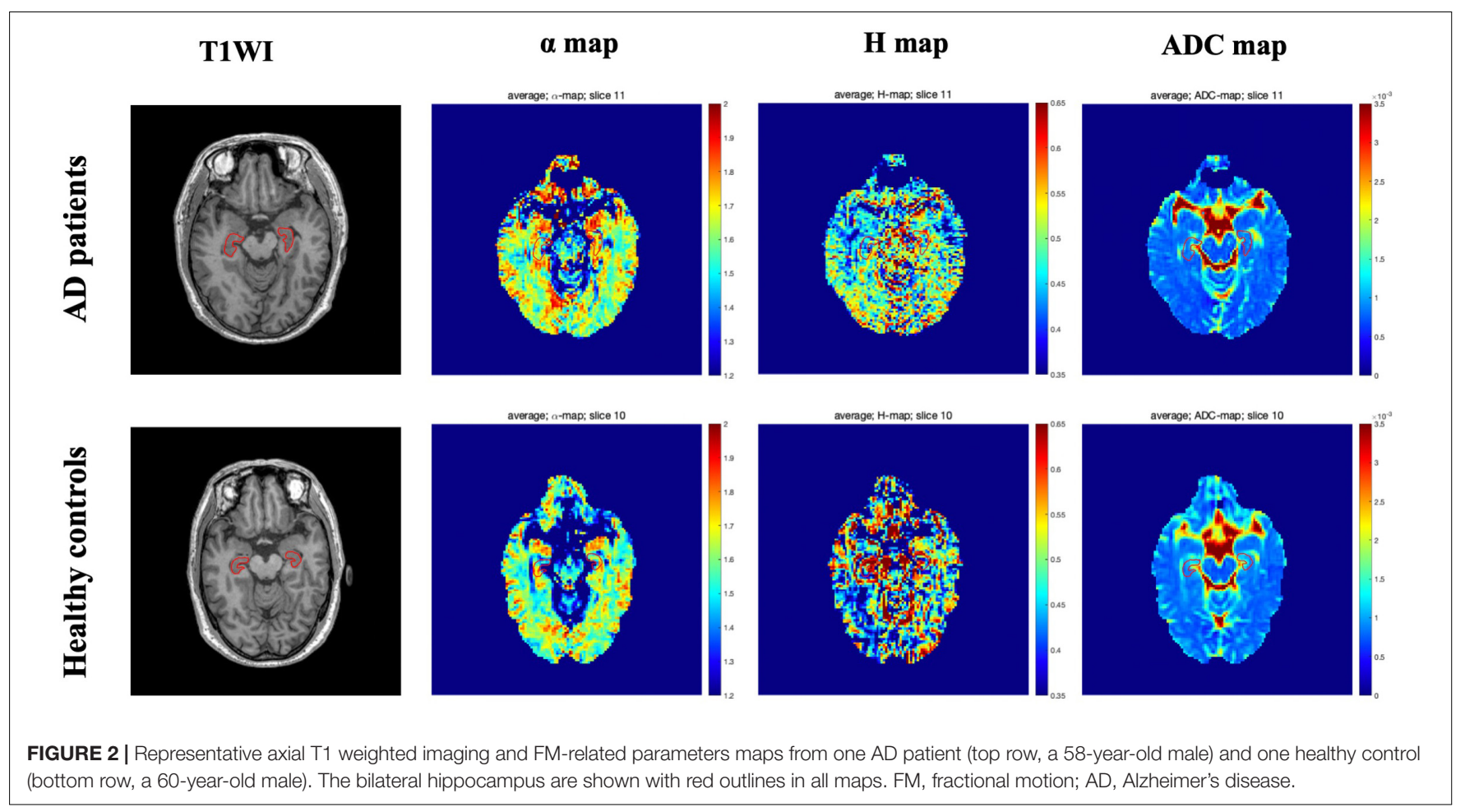

Then the mean values of $\alpha, H, \mu$, and ADC in the bilateral hippocampus were acquired.

\section{Image Analysis}

FSL tools were used to correct head motion and eddy current distortions of the obtained images (Cha, 2006). Apparent diffusion coefficient maps were calculated using the images acquired at $b$-values of 0 and $954 \mathrm{~s} / \mathrm{mm}^{2}$. The images were analyzed using the FM model in order to estimate the anomalous diffusion parameters. According to the dMRI theory based on the FM model (Sui et al., 2015), the diffusion-induced signal decay can be calculated as

$$
S / S_{0}=\exp \left(-\eta D_{\alpha, H} \gamma^{\alpha} G_{0}^{\alpha} \Delta^{\alpha+\alpha H}\right)
$$

where $D_{\alpha, H}$ refers to the diffusion coefficient of anomalous diffusion, and $\gamma$ refers to the gyromagnetic ratio. $G_{0}$ refers to the diffusion gradient amplitude, and $\Delta$ refers to the gradient separation time. $\eta$ refers to a dimensionless number, which is determined by $\alpha, H, \delta$, and $\Delta$ (Xu et al., 2017b, 2018). All fitting procedures were carried out by the trustregion-reflective nonlinear fitting algorithm in MATLAB (MathWorks, Natick, MA).

\section{Statistical Analysis}

The age, education, and MMSE score were compared using OneWay ANOVA among mild AD patients, moderate AD patients, and healthy controls. And their data were shown as mean $\pm \mathrm{SD}$. Gender was compared using the Chi-square $\left(\chi^{2}\right)$ test. $P$ values $<0.05$ were considered statistically significant.
To investigate the potential feasibility of the FM model for distinguishing $\mathrm{AD}$ patients from healthy controls, the mean values of $\alpha, H, \mu$, and $\mathrm{ADC}$ were compared between $\mathrm{AD}$ patients and healthy controls using a two-sample $t$-test. Then these values were also used to identify mild AD and moderate $\mathrm{AD}$ patients using a two-sample $t$-test. Besides, in order to quantify the sensitivity and specificity of $\alpha, H, \mu$, and ADC values in differentiating patients with $\mathrm{AD}$ from healthy controls and distinguishing mild $\mathrm{AD}$ patients and moderate $\mathrm{AD}$ patients, we generated receiver-operating characteristic (ROC) curves and assessed their area under the curve (AUC). The correlations between $\alpha, H, \mu$, and $\mathrm{ADC}$ values and the cognitive functions evaluated by the MMSE and MoCA scales were investigated using Pearson partial correlation analysis in patients with AD.

\section{RESULTS}

\section{Characteristics of All the Subjects}

The demographic and clinical test results of all participants are summarized in Table 1. A total of 24 AD patients ( 9 males and 15 females, mean age $68.96 \pm 8.08$ years) and 11 healthy controls ( 2 males and 9 females, mean age $65.27 \pm 6.60$ years, range 54-78 years, education $10.64 \pm 3.30$ years) were included in this study. According to the MMSE score and education level, $\mathrm{AD}$ patients were divided into a mild $\mathrm{AD}$ group (6 males and 6 females, mean age $65.83 \pm 10.06$ years, range 50-77 years, education $13.42 \pm 3.06$ years) and a moderate $\mathrm{AD}$ group ( 3 males and 9 females, mean age $72.08 \pm 3.75$ years, range $67-79$ years, education $10.50 \pm 3.87$ years). There was no significant difference in the age and education among the three groups $(P>0.05)$. 
While there was a significant difference in the MMSE score among the three groups $(P<0.05)$. And there was a significant difference in the MoCA score between the mild AD group and the moderate $\mathrm{AD}$ group $(P<0.05)$ since a MoCA scale was not collected in the healthy group.

Figure 1 showed the bilateral hippocampus in Axial MRI. Representative maps of the $\mathrm{AD}$ group and control group are shown in Figure 2, showing the $\alpha, H$, and ADC maps and T1weighted images. We can see that there were no obvious image contrasts visible by the naked eye in the $\alpha, H$, and ADC maps in the bilateral hippocampus.

\section{Comparisons of FM-Related Parameters and ADC Values Between AD Patients and Healthy Controls, and Between Mild and Moderate AD Patients}

The mean \pm SD values of the FM-related parameters and ADC values of the bilateral hippocampus in all subjects are summarized in Table 2. Figures 3, 4 show scatter plots to distinguish $\mathrm{AD}$ patients from healthy controls and to differentiate mild $\mathrm{AD}$ and moderate $\mathrm{AD}$ patients, separately. As shown in Figure 3, the AD patients and healthy controls can be readily separated using $\alpha$ (left ROI, $P$-value $=0.011$; right ROI, $P$-value $=0.001$ ) and ADC (left ROI, $P$-value $=0.001$; right ROI, $\mathrm{P}$-value $=0.001)$. Moreover, Figure 4 also shows the identification between mild and moderate AD patients based on FM-related parameters $\alpha$ (right ROI, $P$-value $=0.015)$ and ADC (left ROI, $P$-value $=0.011$; right ROI, $P$-value $=0.022$ ), whereas $H$ and $\mu$ (both $P$-value $>0.05)$ failed to differentiate the two groups.

Receiver-operating characteristic analysis also showed the performance in differentiating patients with $\mathrm{AD}$ from healthy controls. Figure 5 shows the ROC curves calculated from the mean values. Although the number of subjects are limited, the combination of $\alpha$ and ADC (AUC $=0.848$ left ROI, $\mathrm{AUC}=0.856$ right $\mathrm{ROI}$ ) showed an improved performance in differentiating $\mathrm{AD}$ patients and healthy controls as compared with $\alpha(\mathrm{AUC}=0.78$ left $\mathrm{ROI}, \mathrm{AUC}=0.811$ right $\mathrm{ROI})$ or $\mathrm{ADC}$ (AUC $=0.847$ left ROI, AUC $=0.833$ right ROI) alone; when the threshold for $\alpha$ was 1.5939 (left ROI) and 1.58415 (right ROI), the highest Youden index (sum of sensitivity and specificity minus one) showed better sensibility and specificity (left ROI,
0.917 and 0.636; right ROI, 0.917 and 0.727). Similarly, towards differentiating mild and moderate $\mathrm{AD}$ patients (Figure 6), larger AUCs were obtained with $\alpha+$ ADC (AUC $=0.861$ left ROI, $\mathrm{AUC}=0.868$ right $\mathrm{ROI})$ compared with $\alpha(\mathrm{AUC}=0.813$ right $\mathrm{ROI}$ ) or $\mathrm{ADC}$ ( $\mathrm{AUC}=0.792$ left $\mathrm{ROI}$, $\mathrm{AUC}=0.806$ right ROI) alone; when $\alpha=1.55115$ (right ROI), the highest Youden index occurred (sensibility and specificity, right ROI, 0.917 and 0.75). The classification performance of $\alpha+\mathrm{ADC}+H+\mu$ is similar to $\alpha+\mathrm{ADC}$ in differentiating $\mathrm{AD}$ patients and healthy controls (AUC $=0.848$ left $\mathrm{ROI}, \mathrm{AUC}=0.860$ right $\mathrm{ROI}$ ), and grading mild and moderate $\mathrm{AD}$ patients $(\mathrm{AUC}=0.847$ left $\mathrm{ROI}$, $\mathrm{AUC}=0.868$ right $\mathrm{ROI}$ ).

\section{Correlations Between FM-Related Parameters and MMSE Scores and MoCA Scores}

The FM-related parameter $\alpha$ was found to be positively correlated with the MMSE score $(P<0.05$; Figure 7$)$ and MoCA score $(P<0.05$; Figure 8) in patients with AD. No significant correlations were detected in other FM-related parameters and ADC. However, there was no significant correlation between $\alpha$ values and the MMSE score or MoCA score after false discovery rate (FDR) corrections.

\section{DISCUSSION}

The FM model proposed recently is a kind of anomalous diffusion model. In this study, we used the FM model to analyze anomalous diffusion in patients with $\mathrm{AD}$ and healthy controls in vivo. The FM-related parameter maps showed there were no obvious image contrasts visible with the naked eye in the hippocampus (Figure 2). However, the values of their anomalous diffusion parameters could be used to distinguish $\mathrm{AD}$ patients from healthy controls and to identify mild $\mathrm{AD}$ and moderate $\mathrm{AD}$ patients, in particular the $\alpha$. The $\alpha$ provides better sensitivity and specificity for differentiating $\mathrm{AD}$ patients from healthy controls and grading mild $\mathrm{AD}$ and moderate $\mathrm{AD}$ patients. The possible explanations for this are as follows. Autopsies demonstrate the pathological changes of $\mathrm{AD}$ with significantly decreased numbers of neurons, atrophy of residual nerve cells, and varying degrees of degeneration. The characteristic pathological changes of $\mathrm{AD}$, such as $\mathrm{A} \beta$ protein and

TABLE 2 | Mean and SD of the FM-related parameters and ADC values of bilateral hippocampus in mild and moderate AD patients and healthy controls.

\begin{tabular}{|c|c|c|c|c|c|c|}
\hline \multirow[t]{2}{*}{ Subject } & \multirow[t]{2}{*}{ No. } & \multirow[t]{2}{*}{ ROls } & \multicolumn{3}{|c|}{ FM-related parameters } & \multirow[t]{2}{*}{ ADC } \\
\hline & & & $\alpha$ & $\mathbf{H}$ & $\mu$ & \\
\hline \multirow[t]{2}{*}{ Controls } & 11 & Left-hippocampus & $1.588 \pm 0.047$ & $0.464 \pm 0.033$ & $-0.166 \pm 0.024$ & $0.00108 \pm 0.00013$ \\
\hline & & Right-hippocampus & $1.604 \pm 0.049$ & $0.479 \pm 0.028$ & $-0.145 \pm 0.027$ & $0.00104 \pm 0.00012$ \\
\hline \multirow[t]{2}{*}{ Mild AD } & 12 & Left-hippocampus & $1.563 \pm 0.030$ & $0.470 \pm 0.027$ & $-0.170 \pm 0.031$ & $0.00119 \pm 0.00013$ \\
\hline & & Right-hippocampus & $1.562 \pm 0.040$ & $0.485 \pm 0.040$ & $-0.156 \pm 0.045$ & $0.00115 \pm 0.00017$ \\
\hline \multirow[t]{2}{*}{ Moderate AD } & 12 & Left-hippocampus & $1.531 \pm 0.045$ & $0.468 \pm 0.030$ & $-0.186 \pm 0.044$ & $0.00139 \pm 0.00021$ \\
\hline & & Right-hippocampus & $1.519 \pm 0.041$ & $0.481 \pm 0.049$ & $-0.177 \pm 0.050$ & $0.00137 \pm 0.00025$ \\
\hline
\end{tabular}

SD, standard deviation; FM, fractional motion; $A D C$, apparent diffusion coefficient; $A D$, Alzheimer's disease; ROls, region of interest. 

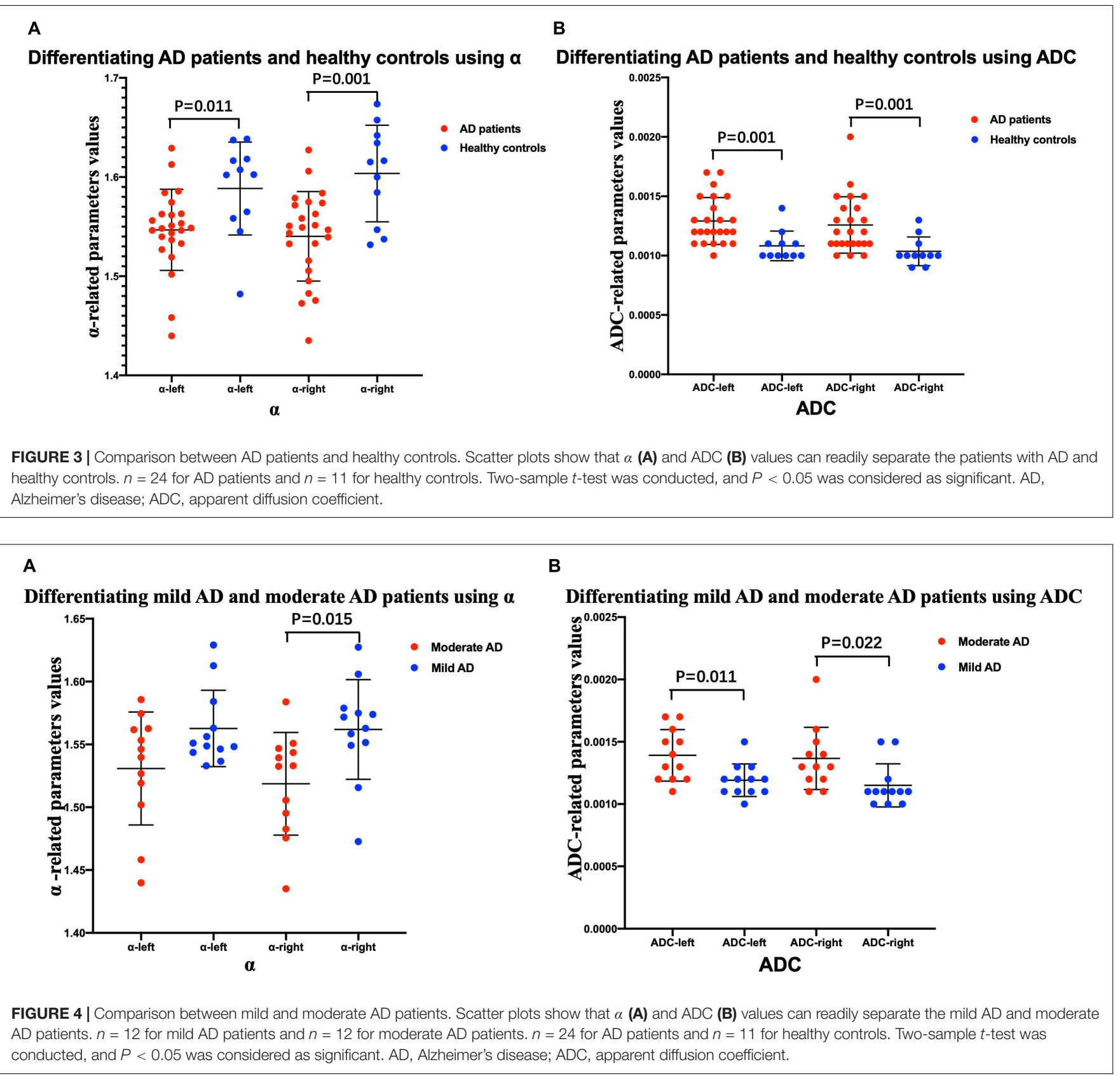

neurofibrillary tangles, can eventually lead to apoptosis (Wang et al., 2015). The progression from normal brain tissue to AD is related to the development of increasing lesions, and the apoptosis and atrophy of neurons, which lead to the reduction of the hippocampal volume. The values of $\alpha$ depend on the structural complexity of the hippocampus, the more complex the hippocampus is, the more significant the non-Gaussian water molecule diffusion, and the greater the $\alpha$ values will be. In theory, the diffusion of water molecules in the brain is affected by many factors, including axons, cell membrane, and myelin (Yoshida et al., 2013). In the disease process of AD, degeneration, atrophy, and apoptosis of neurons lead to a decrease in the complexity of brain tissue, so the $\alpha$ values decrease in this process (Jensen and Helpern, 2010; Grinberg et al., 2011; Yoshida et al., 2013). Therefore, the $\alpha$ values were different between $\mathrm{AD}$ patients and healthy control and between mild $\mathrm{AD}$ and moderate $\mathrm{AD}$ patients.

The FM model has many advantages compared with other MRI theories. Firstly, it is more precise in describing the true diffusion signal decay curve. Secondly, the previous studies have shown that compared with ADC, FM-related parameters are more sensitive and specific in the identification of low-grade and high-grade gliomas ( $\mathrm{Xu}$ et al., 2017b). Thirdly, the data is relatively easy to acquire and the analysis is not complex. The FM model is a potentially better dMRI technology, so we chose the FM model as the research method of AD. 


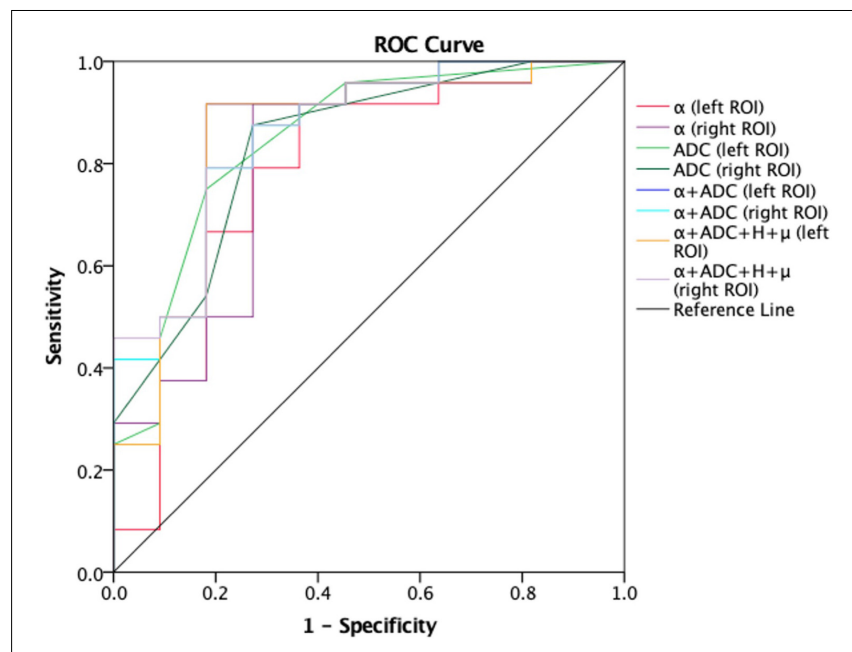

FIGURE 5 | Receiver-operating characteristic (ROC) curves obtained using $\alpha$, ADC, $\alpha+$ ADC, and $\alpha+A D C+\mathrm{H}+\mu$ for differentiating AD patients $(n=24)$ and healthy controls $(n=11)$. The ROC curves were generated using the mean values for subjects. From the figure we can see $\alpha+A D C$ (AUC $=0.848$ left, $\mathrm{AUC}=0.856$ right) is superior to the single $\alpha$ and single ADC.

$\alpha+\mathrm{ADC}+\mathrm{H}+\mu$ and $\alpha+\mathrm{ADC}$ have similar classification performance. ROC, reciever operating characteristic; ADC, apparent diffusion coefficient; AD, Alzheimer's disease; AUC, area under the curve.

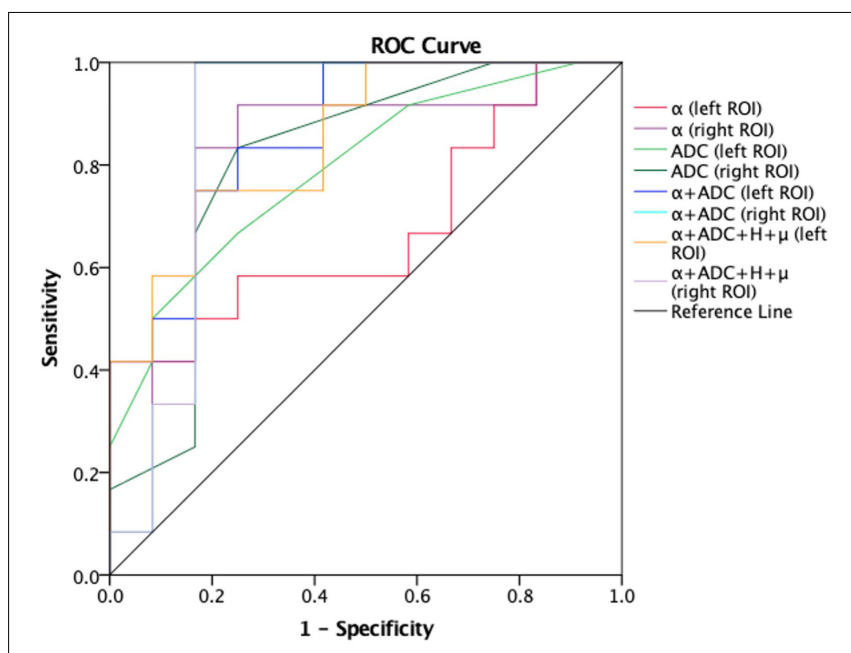

FIGURE 6 | Receiver-operating characteristic (ROC) curves obtained using $\alpha$, ADC, $\alpha+\mathrm{ADC}$, and $\alpha+\mathrm{ADC}+\mathrm{H}+\mu$ differentiating mild AD patients $(n=12)$ and moderate AD patients $(n=12)$. The ROC curves were generated using the mean values for subjects. From the figure we can see $\alpha+A D C$ ( $\mathrm{AUC}=0.861$ left, $\mathrm{AUC}=0.868$ right) is superior to the single $\alpha$ and single ADC. $\alpha+\mathrm{ADC}+\mathrm{H}+\mu$ and $\alpha+\mathrm{ADC}$ have similar classification performance. $R O C$, receiver operating characteristic; $A D C$, apparent diffusion coefficient; $A D$, Alzheimer's disease; AUC, area under the curve.

The reasons why we chose the bilateral hippocampus as ROIs are as follows: (1) $\mathrm{AD}$ is characterized by memory loss and cognitive decline, and the hippocampus is the main brain region that is related to learning and human memory, especially longterm memory. (2) The hippocampus is one of the main brain regions that $A \beta$ and the hyperphosphorylation of tau proteins

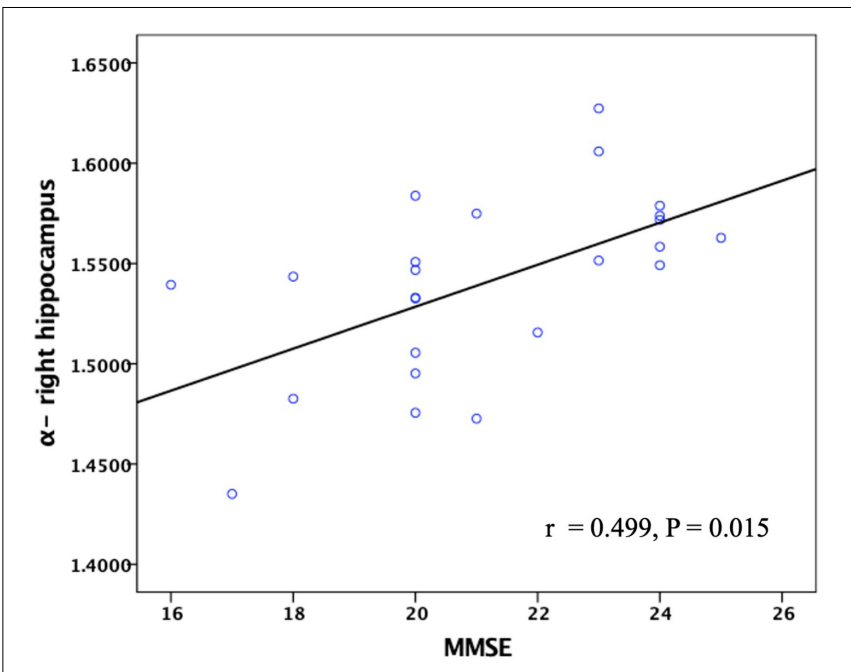

FIGURE 7 | Correlation between the $\alpha$ values of the right hippocampus and MMSE scores in patients with AD. Pearson partial correlation was conducted, $n=24$. MMSE, mini-mental state examination.

(Glenner et al., 1984; Brion et al., 1985; Veitch et al., 2019) are overexpressed, which are the main pathological mechanisms of AD. Besides, many studies found that the internal structure of the hippocampus is asymmetric. And asymmetric expression of proteins and other molecules, and asymmetrical hippocampal morphology have been recently proposed. The functions of the left and right hippocampus are also asymmetrical, the left hippocampus is dominant in the encoding and information transfer stage, and the right hippocampus is dominant in the memory compensation stage (Song et al., 2019). So we compared them separately in this study (Acosta-Cabronero et al., 2013; Du et al., 2018).

Diffusion magnetic resonance imaging investigates the diffusion process at the cellular scale in brain tissues. Different from the mono-exponential model, FM models displayed a better agreement between the experimentally measured signal decay curve and the fitted curves (Magin et al., 2008). From Eq. 1 we can see, the $\alpha$ is an exponent of the diffusion gradient proportional to the parameters offered by other dMRI models, such as the stretching parameter in the stretched exponential model (Bennett et al., 2003; Hall and Barrick, 2008; Zhou et al., 2010). It is reasonable to generalize our finding of $\alpha$ to these parameters. Previous research has shown that the parameters calculated by this method are reliable. And the $\alpha$ refers to the variances of increments of diffusion processes based on the FM theory when the parameter has been interpreted as an index of heterogeneity of water diffusion (Fan and Gao, 2015). It should be admitted that the acquisition and calculation will be largely simplified if only this kind of parameters is of interest. This simplification will reduce the scan time and improve the availability. It is worth mentioning that although $H$ and $\mu$ of the FM model showed no significance in this research, they do provide distinctive information and may be useful if fully explored (Xu et al., 2018). 

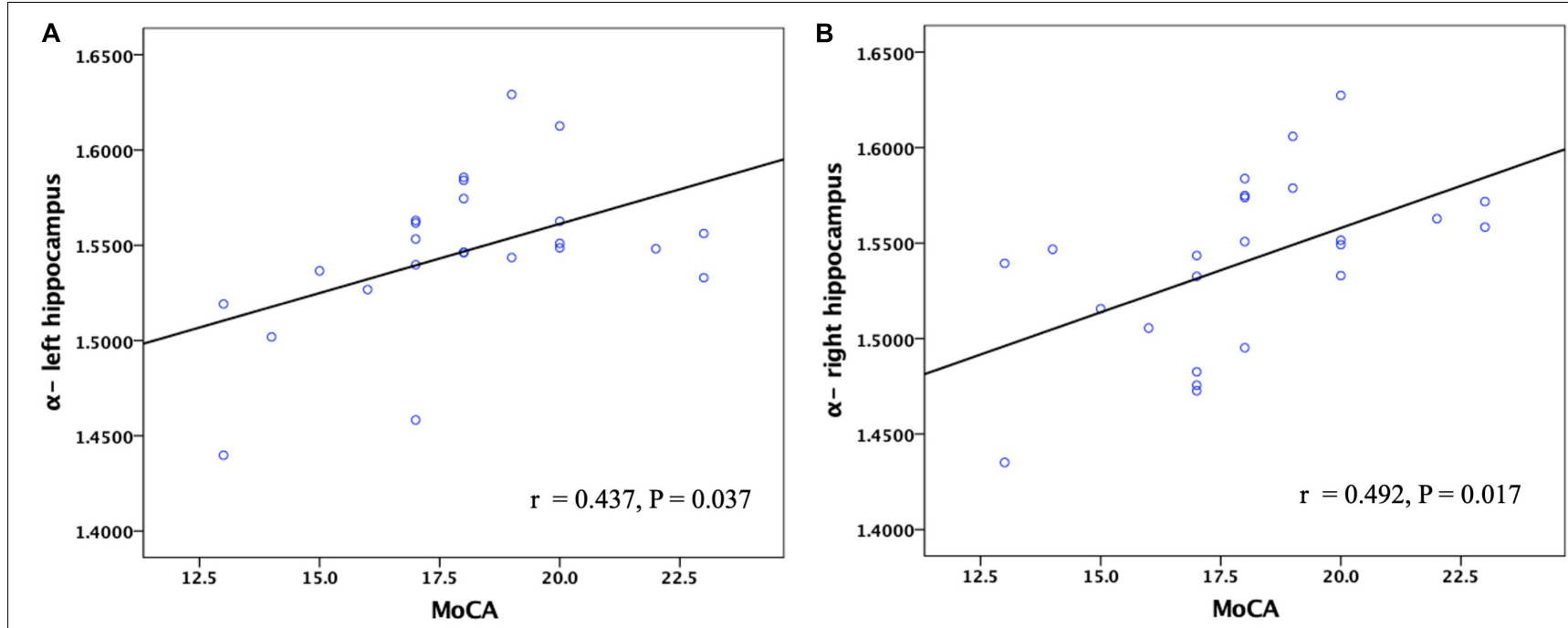

FIGURE 8 | Corelation between the $\alpha$ values of the left and right hippocampus and MoCA scores in patients with AD. Pearson partial correlation was conducted. $n=24$. MoCA, Montreal cognitive assessment.

In terms of identifying $\mathrm{AD}$ patients from healthy controls, the $\mathrm{ADC}$ values in the bilateral hippocampus of our $\mathrm{AD}$ patients were higher than those in healthy controls. These findings were similar to the results of other teams (Kantarci et al., 2001; Petersen, 2004; Zimny et al., 2013). We also found that the $\alpha$ value of our $\mathrm{AD}$ group was lower than that of the healthy control group. The $P$-values are close in $\alpha$ and traditional $\mathrm{ADC}$. In a previous research, the ADC, ultra-high $b$-values ADC (ADC_uh), and diffusion kurtosis imaging (DKI) were used for $\mathrm{AD}$ identification. The AUC of a single parameter was $0.766-0.847$, and the AUC of a combination of all three parameters was 0.868 (Xue et al., 2019). In our study, the ADC and FM model independently showed a similar ability of identification. When they were combined, the AUC value increased, indicating that the $\alpha$ was useful and can significantly improve the diagnostic capability of ADC when combining the $\alpha$ and the ADC.

Similarly, in terms of differentiating mild AD and moderate $\mathrm{AD}$ patients, the $\mathrm{ADC}$ value in the bilateral hippocampus of moderate $\mathrm{AD}$ patients was higher than that of mild $\mathrm{AD}$ patients, and the $\alpha$ value of moderate $\mathrm{AD}$ patients was lower than that of mild AD patients. Besides, the $\alpha$ can also significantly increase the diagnostic capability of $\mathrm{ADC}$ when distinguishing mild $\mathrm{AD}$ and moderate $\mathrm{AD}$ patients. Therefore, the $\alpha$ is very valuable in the research of differentiating and grading $\mathrm{AD}$ patients. In further research, the FM model combined with ADC can greatly improve the diagnostic capability of AD.

Age was an important risk factor that may affect the diffusion process of water in brain tissues and the cognitive function of $\mathrm{AD}$ patients. In order to eliminate the influence of age, Pearson partial correlation analysis was used to compare the relationship between the values of FM-related parameters and MMSE or MoCA scores. And we found that the $\alpha$ values of the hippocampus were positively correlated with MMSE and MoCA scores in the $\mathrm{AD}$ patients, while there were no significant correlations in ADC values. The explanation may be as follows. The values of $\alpha$ depend on the structural complexity of the hippocampus, the more complex the hippocampus is, the greater the $\alpha$ values will be. In the disease process of $\mathrm{AD}$, degeneration, atrophy, and apoptosis of neurons lead to a decrease in complexity of the brain tissue (Jensen and Helpern, 2010; Grinberg et al., 2011), so the $\alpha$ values decrease in this process. It is well known that the cognitive function of $\mathrm{AD}$ patients is gradually declining. Therefore, the $\alpha$ values of the hippocampus were positively correlated with cognitive measures. These findings indicate that the $\alpha$ values of the hippocampus, better than traditional ADC values, might become a potential MRI-based biomarker for disease severity in the mild and moderate $\mathrm{AD}$ patients. However, there was no significant correlation between $\alpha$ values and the MMSE score and MoCA score after FDR correction, indicating that their correlation is not so strong. A possible reason for this was that the sample size was small.

This study has some limitations. Firstly, the number of $\mathrm{AD}$ patients and healthy controls in the present study is limited, so further research with a larger sample size is required to validate our results. Secondly, we only targeted patients with $\mathrm{AD}$ and healthy controls, the inclusion of patients with MCI in further studies is more beneficial for clinical use, since $\mathrm{MCI}$ is considered an early stage of $\mathrm{AD}$ (Petersen, 2004). Thirdly, the single-shot echo-planar imaging used in this study may lead to signal loss and image distortion. These artifacts still occur although they have been reduced due to the development of high-performance gradients and parallel imaging, so the evaluation of the hippocampus may be confined. Fourthly, the voxel was large, so a single voxel showed an aggregated measure of a large sample size, which may obstruct sensitivity to the tissue occupying a small part of a voxel. Further work is needed to explore the voxel-wise radiologic-pathologic correlation. 


\section{CONCLUSION}

In the present study, we selected the FM model to quantitatively calculate the FM-related parameters $\alpha, H, \mu$, and ADC values of the bilateral hippocampus. Our results showed that $\mathrm{AD}$ patients and healthy controls can be readily separated using the $\alpha$ and $\mathrm{ADC}$, and mild $\mathrm{AD}$ and moderate $\mathrm{AD}$ patients can be also distinguished using the $\alpha$ and ADC. It was worth mentioning that the FM-related parameter $\alpha$ of the hippocampus was positively correlated with the cognitive function assessed by MMSE and MoCA scales in the AD patients group, while there was no correlation in $\mathrm{ADC}$ values, indicating that the $\alpha$ in the bilateral hippocampus might be a potential MRIbased biomarker of disease severity in patients with AD. This new diffusion model might be useful for further understanding neuropathologic changes in patients with $\mathrm{AD}$.

\section{DATA AVAILABILITY STATEMENT}

All datasets presented in this study are included in the article/ supplementary material.

\section{ETHICS STATEMENT}

The studies involving human participants were reviewed and approved by Ethics Committee of China-Japan Friendship

\section{REFERENCES}

Acosta-Cabronero, J., Williams, G. B., Cardenas-Blanco, A., Arnold, R. J., Lupson, V., and Nestor, P. J. (2013). In vivo quantitative susceptibility mapping (QSM) in Alzheimer's disease. PLoS One 8:e81093. doi: 10.1371/journal.pone.0081093

Aisen, P. S., Cummings, J., Jack, C. R. Jr., Morris, J. C., and Sperling, R. (2017). On the path to 2025: understanding the Alzheimer's disease continuum. Alzheimers Res. Ther. 9:60. doi: 10.1186/s13195-017-0283-5

Alzheimer's, Association. (2016). 2016 Alzheimer's disease facts and figures. Alzheimers Dement. 12, 459-509. doi: 10.1016/j.jalz.2016.03.001

Beaulieu, C. (2002). The basis of anisotropic water diffusion in the nervous system - a technical review. NMR Biomed. 15, 435-455. doi: 10.1002/nbm.782

Bennett, K. M., Schmainda, K. M., Bennett, R. T., Rowe, D. B., Lu, H., and Hyde, J. S. (2003). Characterization of continuously distributed cortical water diffusion rates with a stretched-exponential model. Magn. Reson. Med. 50, 727-734. doi: 10.1002/mrm.10581

Bosch, B., Arenaza-Urquijo, E. M., Rami, L., Sala-Llonch, R., Junque, C., SolePadulles, C., et al. (2012). Multiple DTI index analysis in normal aging, amnestic MCI and AD. Relationship with neuropsychological performance. Neurobiol. Aging 33, 61-74. doi: 10.1016/j.neurobiolaging.2010.02.004

Braak, H., and Braak, E. (1991). Neuropathological stageing of Alzheimer-related changes. Acta Neuropathol. 82, 239-259. doi: 10.1007/bf00308809

Brion, J. P., Couck, A. M., Passareiro, E., and Flament-Durand, J. (1985). Neurofibrillary tangles of Alzheimer's disease: an immunohistochemical study. J. Submicrosc. Cytol. 17, 89-96.

Bulakbasi, N., Guvenc, I., Onguru, O., Erdogan, E., Tayfun, C., and Ucoz, T. (2004). The added value of the apparent diffusion coefficient calculation to magnetic resonance imaging in the differentiation and grading of malignant brain tumors. J. Comput. Assist. Tomogr. 28, 735-746. doi: 10.1097/00004728200411000-00003

Burnecki, K., and Weron, A. (2010). Fractional Levy stable motion can model subdiffusive dynamics. Phys. Rev. E. Stat. Nonlin. Soft. Matter. Phys. 82:021130. doi: 10.1103/PhysRevE.82.021130
Hospital. The patients/participants provided their written informed consent to participate in this study. Written informed consent was obtained from the individual(s) for the publication of any potentially identifiable images or data included in this article.

\section{AUTHOR CONTRIBUTIONS}

LD, BX, and ZZ analyzed and explained the data and drafted and revised the manuscript. LD and GM designed the study. WG, XH, SSh, XL, YC, YW, and SSu acquired data. BX, JG, and LZ revised the manuscript about FM model theory. All authors approved the final manuscript.

\section{FUNDING}

This study was supported by the National Key Research and Development Program of China (Nos. 2020YFC2003903, 2019YFC0120903, and 2016YFC1307001) and the grants from National Natural Science Foundation of China (NSFC) (Nos. 81971585, 81571641, and 91959123).

\section{ACKNOWLEDGMENTS}

We thank all the subjects who participated in our study.

Cha, S. (2006). Update on brain tumor imaging: from anatomy to physiology. AJNR. Am. J, Neuroradiol. 27, 475-487.

Chenevert, T. L., Brunberg, J. A., and Pipe, J. G. (1990). Anisotropic diffusion in human white matter: demonstration with MR techniques in vivo. Radiology 177, 401-405. doi: 10.1148/radiology.177.2.2217776

Cummings, J. (2017). Disease modification and Neuroprotection in neurodegenerative disorders. Transl. Neurodegener. 6:25. doi: 10.1186/ s40035-017-0096-2

De Santis, S., Gabrielli, A., Palombo, M., Maraviglia, B., and Capuani, S. (2011). Non-Gaussian diffusion imaging: a brief practical review. Magn. Reson. Imaging 29, 1410-1416. doi: 10.1016/j.mri.2011.04.006

Du, L., Zhao, Z., Cui, A., Zhu, Y., Zhang, L., Liu, J., et al. (2018). Increased Iron Deposition on Brain Quantitative Susceptibility Mapping Correlates with Decreased Cognitive Function in Alzheimer's Disease. ACS. Chem. Neurosci. 9, 1849-1857. doi: 10.1021/acschemneuro.8b00194

Fan, Y., and Gao, J. H. (2015). Fractional motion model for characterization of anomalous diffusion from NMR signals. Phys. Rev. E. Stat. Nonlin. Soft. Matter. Phys. 92:012707. doi: 10.1103/PhysRevE.92.012707

Fieremans, E., Burcaw, L. M., Lee, H. H., Lemberskiy, G., Veraart, J., and Novikov, D. S. (2016). In vivo observation and biophysical interpretation of timedependent diffusion in human white matter. Neuroimage 129, 414-427. doi: 10.1016/j.neuroimage.2016.01.018

Glenner, G. G., Wong, C. W., Quaranta, V., and Eanes, E. D. (1984). The amyloid deposits in Alzheimer's disease: their nature and pathogenesis. Appl. Pathol. 2, 357-369.

Grinberg, F., Farrher, E., Kaffanke, J., Oros-Peusquens, A. M., and Shah, N. J. (2011). Non-Gaussian diffusion in human brain tissue at high b-factors as examined by a combined diffusion kurtosis and biexponential diffusion tensor analysis. Neuroimage 57, 1087-1102. doi: 10.1016/j.neuroimage.2011. 04.050

Hall, M. G., and Barrick, T. R. (2008). From diffusion-weighted MRI to anomalous diffusion imaging. Magn. Reson. Med. 59, 447-455. doi: 10.1002/mrm. 21453 
Hyman, B. T., Van Hoesen, G. W., Damasio, A. R., and Barnes, C. L. (1984). Alzheimer's disease: cell-specific pathology isolates the hippocampal formation. Science 225, 1168-1170. doi: 10.1126/science.6474172

Jensen, J. H., and Helpern, J. A. (2010). MRI quantification of non-Gaussian water diffusion by kurtosis analysis. NMR. Biomed. 23, 698-710. doi: 10.1002/nbm. 1518

Jensen, J. H., Helpern, J. A., Ramani, A., Lu, H., and Kaczynski, K. (2005). Diffusional kurtosis imaging: the quantification of non-gaussian water diffusion by means of magnetic resonance imaging. Magn. Reson. Med. 53, 1432-1440. doi: $10.1002 / \mathrm{mrm} .20508$

Kantarci, K., Jack, C. R. Jr., Xu, Y. C., Campeau, N. G., and O’Brien, P. C. (2001). Mild cognitive impairment and Alzheimer disease: regional diffusivity of water. Radiology 219, 101-107. doi: 10.1148/radiology.219.1.r01ap14101

Khan, A., Corbett, A., and Ballard, C. (2017). Emerging treatments for Alzheimer's disease for non-amyloid and non-tau targets. Expert Rev. Neurother. 17, 683695. doi: 10.1080/14737175.2017.1326818

Kidd, M. (1963). Paired helical filaments in electron microscopy of Alzheimer's disease. Nature 197, 192-193. doi: 10.1038/197192b0

Le Bihan, D., and Johansen-Berg, H. (2012). Diffusion MRI at 25: exploring brain tissue structure and function. Neuroimage 61, 324-341. doi: 10.1016/j. neuroimage.2011.11.006

Magdziarz, M., Weron, A., Burnecki, K., and Klafter, J. (2009). Fractional brownian motion versus the continuous-time random walk: a simple test for subdiffusive dynamics. Phys. Rev. Lett. 103:180602. doi: 10.1103/PhysRevLett.103.180602

Magin, R. L., Abdullah, O., Baleanu, D., and Zhou, X. J. (2008). Anomalous diffusion expressed through fractional order differential operators in the BlochTorrey equation. J. Magn. Reson. 190, 255-270. doi: 10.1016/j.jmr.2007.11. 007

Maier, S. E., Bogner, P., Bajzik, G., Mamata, H., Mamata, Y., Repa, I., et al. (2001). Normal brain and brain tumor: multicomponent apparent diffusion coefficient line scan imaging. Radiology 219, 842-849. doi: 10.1148/radiology. 219.3.r01jn02842

Mayo, C. D., Garcia-Barrera, M. A., Mazerolle, E. L., Ritchie, L. J., Fisk, J. D., Gawryluk, J. R., et al. (2018). Relationship Between DTI Metrics and Cognitive Function in Alzheimer's Disease. Front. Aging Neurosci. 10:436. doi: 10.3389/ fnagi.2018.00436

Moseley, M. E., Cohen, Y., Kucharczyk, J., Mintorovitch, J., Asgari, H. S., Wendland, M. F., et al. (1990). Diffusion-weighted MR imaging of anisotropic water diffusion in cat central nervous system. Radiology 176, 439-445. doi: 10.1148/radiology.176.2.2367658

Mulkern, R. V., Gudbjartsson, H., Westin, C. F., Zengingonul, H. P., Gartner, W., Guttmann, C. R., et al. (1999). Multi-component apparent diffusion coefficients in human brain. NMR Biomed. 12, 51-62. doi: 10.1002/(sici)1099-1492(199902) 12:1<51::aid-nbm546<3.0.co;2-e

Nir, T. M., Jahanshad, N., Villalon-Reina, J. E., Toga, A. W., Jack, C. R., Weiner, M. W., et al. (2013). Effectiveness of regional DTI measures in distinguishing Alzheimer's disease, MCI, and normal aging. Neuroimage Clin. 3, 180-195. doi: 10.1016/j.nicl.2013.07.006

Petersen, R. C. (2004). Mild cognitive impairment as a diagnostic entity. J. Intern. Med. 256, 183-194. doi: 10.1111/j.1365-2796.2004.01388.x

Pietroboni, A. M., Scarioni, M., Carandini, T., Basilico, P., Cadioli, M., Giulietti, G., et al. (2018). CSF beta-amyloid and white matter damage: a new perspective on Alzheimer's disease. J. Neurol. Neurosurg. Psychiatry 89, 352-357. doi: 10.1136/ jnnp-2017-316603

Reddy, P. H., and Oliver, D. M. (2019). Amyloid Beta and Phosphorylated TauInduced Defective Autophagy and Mitophagy in Alzheimer's Disease. Cells 8:488. doi: 10.3390/cells 8050488

Salvatore, C., Cerasa, A., and Castiglioni, I. (2018). MRI Characterizes the Progressive Course of AD and Predicts Conversion to Alzheimer's Dementia 24 Months Before Probable Diagnosis. Front. Aging Neurosci. 10:135. doi: 10. 3389/fnagi.2018.00135

Song, G., Yao, T., Wang, D., and Li, Y. (2019). Differentiating between Alzheimer's disease, amnestic mild cognitive impairment, and normal aging diffusion kurtosis imaging. Neural. Regen Res. 14, 2141-2146. doi: 10.4103/1673-5374. 262594

Sui, Y., Wang, H., Liu, G., Damen, F. W., Wanamaker, C., Li, Y., et al. (2015). Differentiation of Low- and High-Grade Pediatric Brain Tumors with High b-Value Diffusion-weighted MR Imaging and a Fractional
Order Calculus Model. Radiology 277, 489-496. doi: 10.1148/radiol.20151 42156

Veitch, D. P., Weiner, M. W., Aisen, P. S., Beckett, L. A., Cairns, N. J., Green, R. C., et al. (2019). Understanding disease progression and improving Alzheimer's disease clinical trials: Recent highlights from the Alzheimer's Disease Neuroimaging Initiative. Alzheimers Dement 15, 106-152. doi: 10.1016/ j.jalz.2018.08.005

Wang, D., Guo, Z., Liu, X., Li, Y., and Wang, H. (2015). Examination of hippocampal differences between Alzheimer disease, amnestic mild cognitive impairment and normal aging: diffusion kurtosis. Curr. Alzheimer Res. 12, 80-87. doi: 10.2174/1567205012666141218142422

Wegmann, S., Jung, Y. J., Chinnathambi, S., Mandelkow, E. M., Mandelkow, E., and Muller, D. J. (2010). Human Tau isoforms assemble into ribbon-like fibrils that display polymorphic structure and stability. J. Biol. Chem. 285, 27302-27313. doi: 10.1074/jbc.M110.145318

Weiss, M. (2013). Single-particle tracking data reveal anticorrelated fractional Brownian motion in crowded fluids. Phys. Rev. E. Stat. Nonlin. Soft. Matter. Phys. 88:010101. doi: 10.1103/PhysRevE.88.010101

Wortmann, M. (2012). Dementia: a global health priority - highlights from an ADI and World Health Organization report. Alzheimers Res. Ther. 4:40. doi: 10.1186/alzrt143

Xu, B., Gong, G., Fan, Y., Wu, B., and Gao, J. H. (2017a). Directional sensitivity of anomalous diffusion in human brain assessed by tensorial fractional motion model. Magn. Reson. Imaging 42, 74-81. doi: 10.1016/j.mri.2017.05.006

Xu, B., Su, L., Wang, Z., Fan, Y., Gong, G., Zhu, W., et al. (2017b). Anomalous diffusion in cerebral glioma assessed using a fractional motion model. Magn. Reson Med. 78, 1944-1949. doi: 10.1002/mrm.26581

Xu, B., Su, L., Wang, Z., Fan, Y., Gong, G., Zhu, W., et al. (2018). Anisotropy of anomalous diffusion improves the accuracy of differentiating low- and highgrade cerebral gliomas. Magn. Reson Imaging 51, 14-19. doi: 10.1016/j.mri. 2018.04.005

Xue, Y., Zhang, Z., Wen, C., Liu, H., Wang, S., Li, J., et al. (2019). Characterization of Alzheimer's Disease Using Ultra-high b-values Apparent Diffusion Coefficient and Diffusion Kurtosis Imaging. Aging Dis. 10, 1026-1036. doi: 10.14336/AD.2018.1129

Yamasaki, F., Kurisu, K., Satoh, K., Arita, K., Sugiyama, K., Ohtaki, M., et al. (2005). Apparent diffusion coefficient of human brain tumors at MR imaging. Radiology 235, 985-991. doi: 10.1148/radiol.2353031338

Yoshida, S., Oishi, K., Faria, A., and Mori, S. (2013). Diffusion tensor imaging of normal brain development. Pediatr. Radiol. 43, 15-27. doi: 10.1007/s00247012-2496-x

Yoshiura, T., Mihara, F., Tanaka, A., Ogomori, K., Ohyagi, Y., Taniwaki, T., et al. (2003). High b value diffusion-weighted imaging is more sensitive to white matter degeneration in Alzheimer's disease. Neuroimage 20, 413-419. doi: 10. 1016/s1053-8119(03)00342-2

Zhou, X. J., Gao, Q., Abdullah, O., and Magin, R. L. (2010). Studies of anomalous diffusion in the human brain using fractional order calculus. Magn. Reson. Med. 63, 562-569. doi: 10.1002/mrm.22285

Zimny, A., Bladowska, J., Neska, M., Petryszyn, K., Guzinski, M., Szewczyk, P., et al. (2013). Quantitative MR evaluation of atrophy, as well as perfusion and diffusion alterations within hippocampi in patients with Alzheimer's disease and mild cognitive impairment. Med. Sci. Monit. 19, 86-94. doi: 10.12659/msm. 883757

Conflict of Interest: BX was employed by company Beijing Intelligent Brain Cloud Inc.

The remaining authors declare that the research was conducted in the absence of any commercial or financial relationships that could be construed as a potential conflict of interest.

Copyright (C) $2020 \mathrm{Du}, \mathrm{Xu}, \mathrm{Zhao}, \mathrm{Han}, \mathrm{Gao}$, Shi, Liu, Chen, Wang, Sun, Zhang, Gao and Ma. This is an open-access article distributed under the terms of the Creative Commons Attribution License (CC BY). The use, distribution or reproduction in other forums is permitted, provided the original author(s) and the copyright owner(s) are credited and that the original publication in this journal is cited, in accordance with accepted academic practice. No use, distribution or reproduction is permitted which does not comply with these terms. 